\title{
Modulated electro-hyperthermia enhances dendritic cell therapy through an abscopal effect in mice
}

\author{
WEI QIN ${ }^{1}$, YASUNORI AKUTSU ${ }^{1}$, GABOR ANDOCS $^{2}$, AKIKO SUGANAMI $^{3}$, XIN HU $^{1}$, GULBOSTAN YUSUP $^{1}$, \\ AKI KOMATSU-AKIMOTO ${ }^{1}$, ISAMU HOSHINO ${ }^{1}$, NAOYUKI HANARI ${ }^{1}$, MIKITO MORI ${ }^{1}$, \\ YUKA ISOZAKI $^{1}$, NAOKI AKANUMA ${ }^{1}$, YUTAKA TAMURA ${ }^{3}$ and HISAHIRO MATSUBARA ${ }^{1}$ \\ ${ }^{1}$ Department of Frontier Surgery, Graduate School of Medicine, Chiba University, Chiba 260-8670; \\ ${ }^{2}$ Department of Veterinary Clinical Medicine, Faculty of Veterinary Science, Tottori University, Tottoti 680-8553; \\ ${ }^{3}$ Department of Bioinformatics, Graduate School of Medicine, Chiba University, Chiba 260-8670, Japan
}

Received July 2, 2014; Accepted August 25, 2014

DOI: 10.3892/or.2014.3500

\begin{abstract}
The aim of this study was to assess whether modulated electro-hyperthermia (mEHT) can induce an abscopal effect and thereby enhance the antitumor effects of immunotherapy. We used an intratumoral dendritic cell (DC) injection and $\mathrm{mEHT}$ to treat $\mathrm{C} 3 \mathrm{H} / \mathrm{He}$ mice inoculated with squamous cell carcinoma SCCVII cells in the left leg, and we assessed the whole body antitumor effects. Tumors were examined every two or three days in order to assess growth inhibition. The tumor-draining lymph nodes were removed to enable flow cytometric analysis of $\mathrm{CD}^{+}$and $\mathrm{CD} 8^{+}$cells, whereas immunohistochemistry was used to assess CD8, S100 and Foxp3 expression in the tumors. Additionally, GP96 expression in the tumors from the different treatment groups was measured. In the control group, the mean tumor volume was larger than that in other groups. These results indicated that the combination therapy of an intratumoral DC injection and mEHT evoked systemic antitumor activity. A larger number of $\mathrm{CD}^{+}$and $\mathrm{CD} 8^{+}$ cells were detected by flow cytometric analysis in the DC plus $\mathrm{mEHT}$ treatment group. Tumor tissue immunostaining showed that CD8 and S100 were more strongly expressed in the DC plus mEHT treatment group, although Foxp3 expression was much higher in the control group. The GP96 gene expression level in the mEHT group was significantly different from the expression level in the control group. An abscopal effect may be induced by mEHT, and the effect of immunotherapy with DCs was strongly enhanced by the overexpression of GP96. GP96 is thought to be one of the molecules explaining the abscopal effect. Direct intratumoral administration of DCs and mEHT may be a feasible future treatment strategy.
\end{abstract}

Correspondence to: Dr Yasunori Akutsu, Department of Frontier Surgery, Graduate School of Medicine, Chiba University, 1-8-1 Inohana, Chuo-ku, Chiba, 240-8470, Japan

E-mail: yakutsu@faculty.chiba-u.jp

Key words: dendritic cells, hyperthermia, abscopal effect, cancer therapy, immunotherapy

\section{Introduction}

The abscopal effect is a phenomenon noted in the treatment of metastatic cancer where localized irradiation of a tumor causes shrinking not only of the immediate target, but also of tumors located far from the irradiated area. This phenomenon is extremely rare, but when it does occur, its anticancer effects can be dramatic, leading to the disappearance of malignant growths throughout the body. Such success has been described for a variety of malignancies, including melanoma, lymphoma, and lung metastases of hepatocellular carcinoma (1-3). The mechanism underlying the abscopal effect remains to be clarified, although a variety of underlying biological events may be responsible, particularly those induced by the immune system (4). In mouse studies, the observations support the immune hypothesis for the abscopal effect (5-7).

The modulated electro-hyperthermia (mEHT) system is a fast-developing complementary treatment method that is effective against different types of tumors $(8,9)$. The principles involved are based on the classical method of hyperthermia, but the aim, besides the absolute increase in temperature, is to promote the direct absorption of electric-field energy by the extracellular fluid, thereby destroying the membrane of cancer cells.

Hyperthermia is usually applied as an adjunct to an already established treatment modality, such as radiotherapy and chemotherapy, in order to achieve tumor temperatures of $40-46^{\circ} \mathrm{C}$. Hyperthermia was originally controlled through modulating temperature alone, whereas the more recently developed mEHT (also described as 'oncothermia') is based on energy dose control, replacing the single temperature concept (9).

Although hyperthermia has been extensively studied, the effects of mEHT combined with dendritic cell (DC) treatment on squamous cell carcinoma have not been addressed. The goal of this study was to evaluate the antitumor effects of combined direct intratumoral injection of DCs and mEHT treatment using a mouse squamous cell carcinoma (SCCVII) cancer model. We showed that this combined treatment causes both local and distant shrinkage of SCCVII-derived tumors, 
and thereby offers hope to patients with esophageal squamous cell carcinoma, particularly those who have recurrent disease and are unable to undergo further radiotherapy. Thus, this newly developed technique could represent a new strategy for the treatment of squamous cell carcinoma, as well as other cancers.

\section{Materials and methods}

Culture of SCCVII tumor cells. Mouse SCCVII cells, which have been previously characterized (10), were used in this study. Cells were cultured at $37^{\circ} \mathrm{C}$ in Dulbecco's modified Eagle's medium (DMEM; D8062; Sigma Life Science) with $10 \%$ heat-inactivated fetal bovine serum (FBS; 16140-071; Gibco, Invitrogen) plus 1\% glutamine-penicillin-streptomycin.

DC generation. DCs were generated using the method established by Lutz et al (11). Cells were prepared from the bone marrow cells of the femurs and tibias of mice. On day $0,2 \times 10^{6}$ bone marrow cells in $10 \mathrm{ml}$ of RPMI-1640 medium (RPMI, 22400-089; Gibco, Invitrogen) with penicillin (100 U/ml), streptomycin $(100 \mu \mathrm{g} / \mathrm{ml})$, L-glutamine $(2 \mathrm{mM}), 2$-mercaptoethanol $(50 \mu \mathrm{M}), 10 \% \mathrm{FBS}$ and $200 \mathrm{U} / \mathrm{ml}(20 \mathrm{ng} / \mathrm{ml})$ rmGM-CSF (G0282; Sigma, Japan) were placed into 100-mm diameter Petri dishes $\left(\right.$ Falcon $^{\mathrm{TM}}$ no. 1029; BD Biosciences, San Jose, CA, USA). On day 3, another $10 \mathrm{ml}$ of the medium with supplements was added to the dishes. Non-adherent cells were harvested on day 6 .

We determined whether the cells generated could be used as DCs by performing flow cytometry analysis. Surface markers such as MHC class II, CD11c, CD80 and CD86 were highly expressed by $70.6,35.2,63.4$, and $72.0 \%$ of the cells, respectively, indicating that they were indeed DCs.

Mice and the tumor model. Syngeneic 6- to 10-week-old female $\mathrm{C} 3 \mathrm{H} / \mathrm{He}$ mice, purchased from Japan SLC (Shizuoka, Japan), were maintained in our facility under specific pathogen-free conditions. SCCVII cancer cells at a dose of $5 \times 10^{5}$ per mouse were inoculated subcutaneously into the left leg in order to seed the primary treatment tumor, and the same number of SCCVII cells was simultaneously inoculated subcutaneously into the chest wall to seed a distant, non-treatment tumor. Mice were examined every two or three days, and only those of a similar size were selected for treatment. Animals that had developed palpable tumors 9 days after inoculation prepared for treatment were divided into independent experimental groups, each consisting of at least 4 mice. Animal care was provided in accordance with the guidelines of Chiba University. All animal experiments were conducted to the Guidelines for the Welfare and Use of Animals in Cancer Research (12).

$m E H T$ and DC treatment. We used the LAB-EHY device (Oncotherm Ltd. Hungary) to induce mEHT treatment on the left leg tumors of the mice. The RF power level was regulated using the fluoroptic temperature measurement system (Luxtron m3300; Lumasense, Santa Clara, CA, USA). Tumors on the left legs of the mice in the mEHT-only treatment group and in the DC combined with mEHT treatment group were treated on day 9,11 and 13 after SCCVII cell inoculation. In the DC-only treatment group and DC combined with mEHT treatment

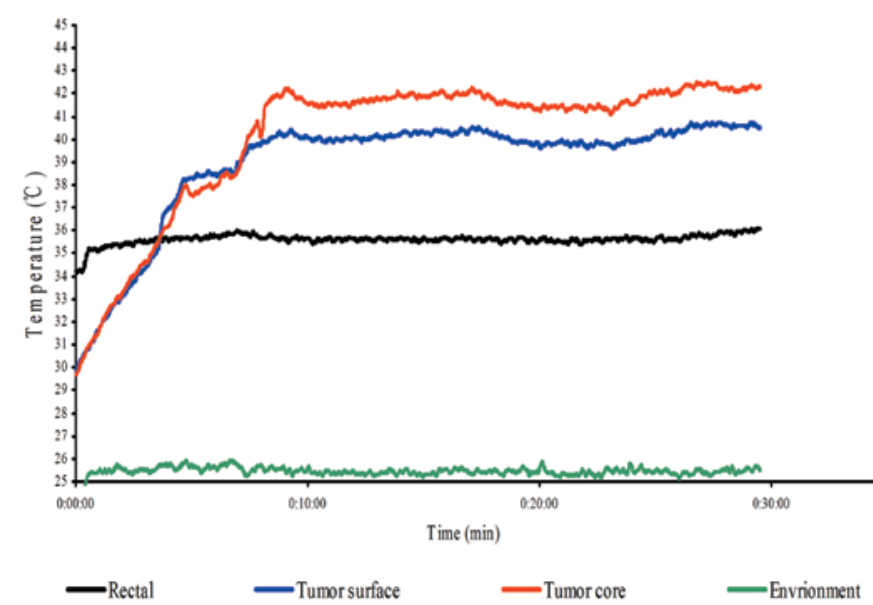

Figure 1. The electric current of the alternating magnetic fields was controlled and the temperature of the thermoseeds was maintained within a range of $42-43^{\circ} \mathrm{C}$. The rectal temperature was maintained at $35-37^{\circ} \mathrm{C}$.

group, $1 \times 10^{6} \mathrm{DCs}$ for each mouse were directly administered only into the tumor on the left leg of the mice after the mEHT treatment on day 9,11 and 13 .

Flow cytometric analysis for cytotoxic T lymphocytes. After the mice were sacrificed on day 39 after SCCVII cell inoculation, the tumor draining lymph nodes (TDLNs) were collected and washed with PBS. After red blood cell reduction, the cells were treated with mouse BD Fc Block (2.4G2; Pharmingen ${ }^{\mathrm{TM}}$, $\mathrm{BD})$ and then stained with antibodies conjugated with fluorescent agents. For the investigation of cytotoxic T lymphocytes (CTLs) in the TDLNs, CD3-PE (2134-0034; Biogenesis Inc., Kingston, NH, USA) and CD8-FITC (2134-0083; Biogenesis Inc.) were used. Cell counting was performed with a Coulter Epics XL cytometer (Beckman Coulter, Miami, FL, USA), and cell populations were evaluated with gating software, FlowJo for Windows (Tree Star Inc., Ashland, OR, USA).

Immunostaining. After the mice were sacrificed on day 39 after SCCVII cell inoculation, the tumor tissues on the chest were harvested. The tissues were stained with anti-mouse CD8 (250596; ABBIOTEC), S100 (GEX48819; Gene Tex), Foxp3 (NBP1-18319; Novus Biologicals) and a fluoresceinconjugated secondary antibody (k4003; Dako). The expression of CD8, S100 and Foxp3 was observed under a microscope (Carl Zeiss, Oberkochen, Germany). According to the method developed by Xavie et al (13), we quantified the results of the immunostaining with the Imag Pro Plus 6.0 software (Media Cybernetic, Silver Spring, MD, USA). The measurement parameter was integrated optical density (IOD). The signal density of the tissue areas from five randomly selected visions were counted blindly and subjected to statistical analysis.

Real-time quantitative PCR (RT-PCR). The RNA was extracted from tumor tissues on the chest using TRIzol reagent (Invitrogen, Carlsbad, CA, USA) and $1 \mu \mathrm{g}$ of RNA was transcribed into cDNA using a High Capacity cDNA Reverse Transcription kit (Applied Biosystems, Foster City, CA, USA). The resulting $1 \mu \mathrm{g}$ of cDNA was amplified via RT-PCR. 

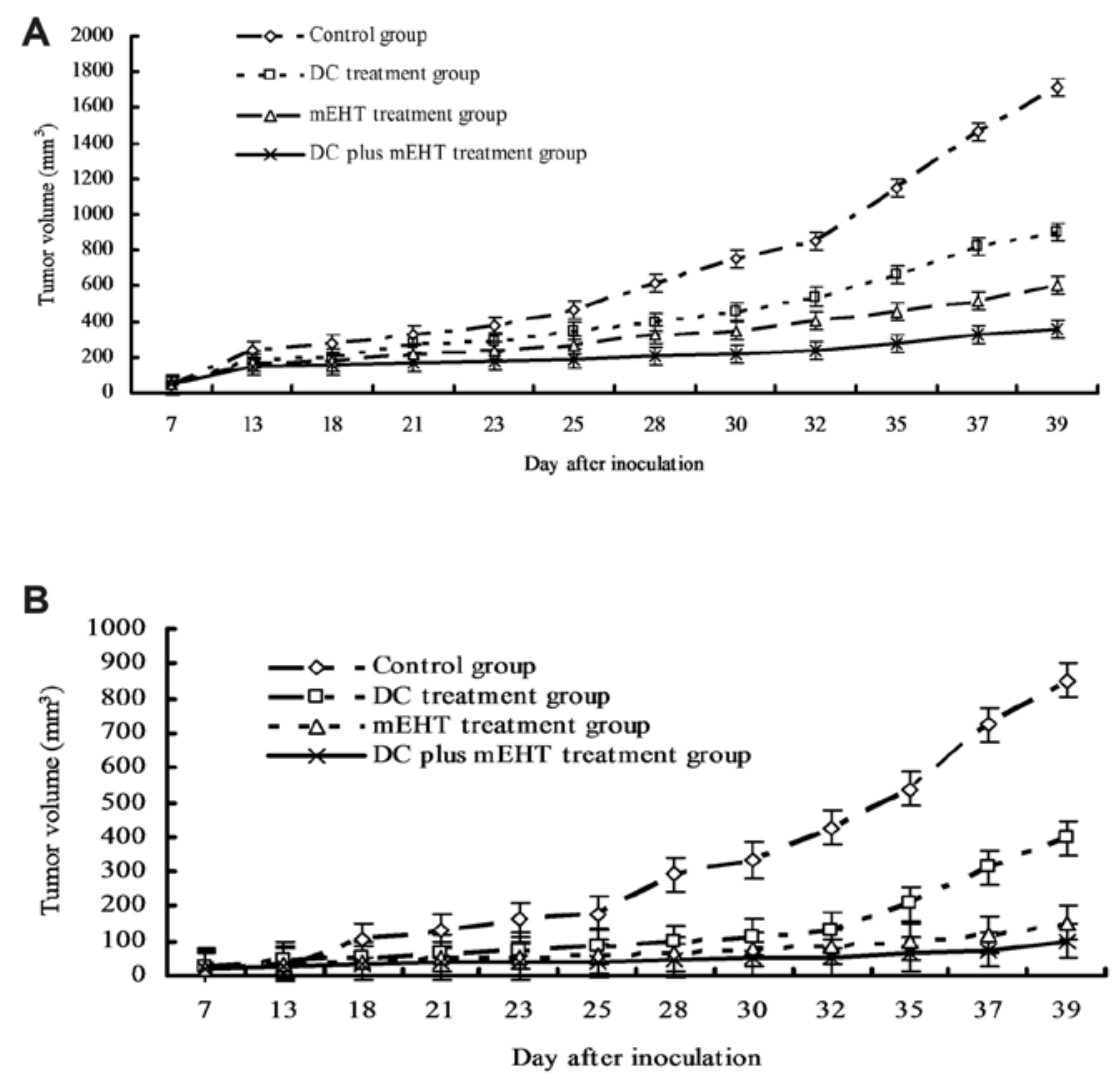

Figure 2. Tumor growth inhibition results. (A) The effectiveness of the combination treatment of dendritic cells (DCs) and modulated electro-hyperthermia (mEHT). The DC plus mEHT treatment completely suppressed the tumor growth on the left leg of the mice. (B) Tumor growth inhibition mediated by the systemic antitumor effect induced by the combination of DCs and mEHT on the tumors without treatment. In these groups, growth of the distant tumor on the chest wall of the mice without treatment was markedly suppressed, compared with mice treated with DCs and mEHT alone.

RT-PCR was conducted using SsoFast ${ }^{\mathrm{TM}}$ EvaGreen $^{\circledR}$ Supermix (Bio-Rad, Hercules, CA, USA) with the following primers (Operon, Biotechnology, Tokyo, Japan): GP96, 5'-ACACGGC TTGCTAAACTTCT-3' and 5'-ACTACAGTCTGCGGTCC AAA-3'. $\beta$-actin was used as an internal control and the sequences used were 5'-TCATGAAGATCCTCACCGAG-3' and 5'-TTGCCAATGGTGATTGACCTG-3'. The PCR was performed using MyiQ2 thermal cycler (Bio-Rad). The PCR conditions consisted of 40 cycles of $95^{\circ} \mathrm{C}$ for $30 \mathrm{sec}, 95^{\circ} \mathrm{C}$ for $5 \mathrm{sec}$ and $60^{\circ} \mathrm{C}$ for $10 \mathrm{sec}$. This assay was performed in triplicate.

Statistics. Results are expressed as the mean \pm SE, and statistical comparisons were performed using ANOVA. A P-value $<0.05$ was considered to indicate a statistically significant result. All data were analyzed by the SPSS software package (SPSS version 19.0; SPSS Inc., USA).

\section{Results}

Temperature monitoring in local mEHT treatment. By using the LAB-EHY device, it was possible to increase the temperature within the tumor to $42^{\circ} \mathrm{C}$ within $10 \mathrm{~min}$. By controlling the energy input, we were able to maintain the temperature within a range of $42-43^{\circ} \mathrm{C}$. At the same time of treatment, we found that the temperature on the surface of the tumor was $\sim 40^{\circ} \mathrm{C}$, and the rectal temperature was maintained close to 35-37 ${ }^{\circ} \mathrm{C}$ (Fig. 1).

Efficacy of the combined therapy of DCs and mEHT against the treated local tumor. We examined the antitumor effect by the combination of DCs and mEHT treatment against the local tumor. The tumor on the left leg of the mice in the control group grew to a mean volume of $1,709.25 \pm 2,5574 \mathrm{~mm}^{3}$ by day 39. The mean volume of the tumor on the left leg in the group treated with a combination of DCs and mEHT was $359.92 \pm 62.01 \mathrm{~mm}^{3}$. The tumor growth rate was obviously slow in the DC and mEHT treatment group compared to that in the control group $(\mathrm{P}<0.05)$. The mean tumor volume in the DC-only treatment group and the mEHT-only treatment group was smaller compared to the control group (DC treatment group: $902.78 \pm 109.74 \mathrm{~mm}^{3}, \mathrm{P}<0.05$; mEHT treatment group: $607.19 \pm 87.28 \mathrm{~mm}^{3}, \mathrm{P}<0.05$ ) (Fig. 2A). Thus, the combination therapy of DCs and mEHT showed the strongest antitumor effect in vivo.

Efficacy of the combined therapy of DCs and mEHT against the non-treated distant tumor. We examined the tumors on the chest wall of the mice to investigate whether the combination treatment of DCs and mEHT could elicit a systemic antitumor effect. On day 39, the mean volume of the tumors on the chest wall of the mice in the control group was $852.43 \pm 270.78 \mathrm{~mm}^{3}$, 
$\mathrm{CD}^{+}$
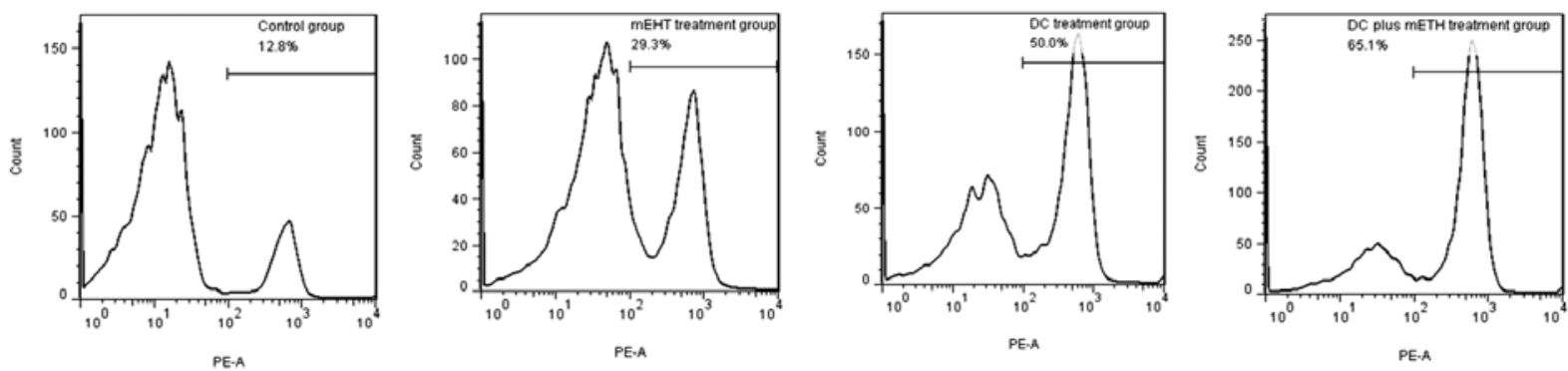

$\mathrm{CD}^{+}$
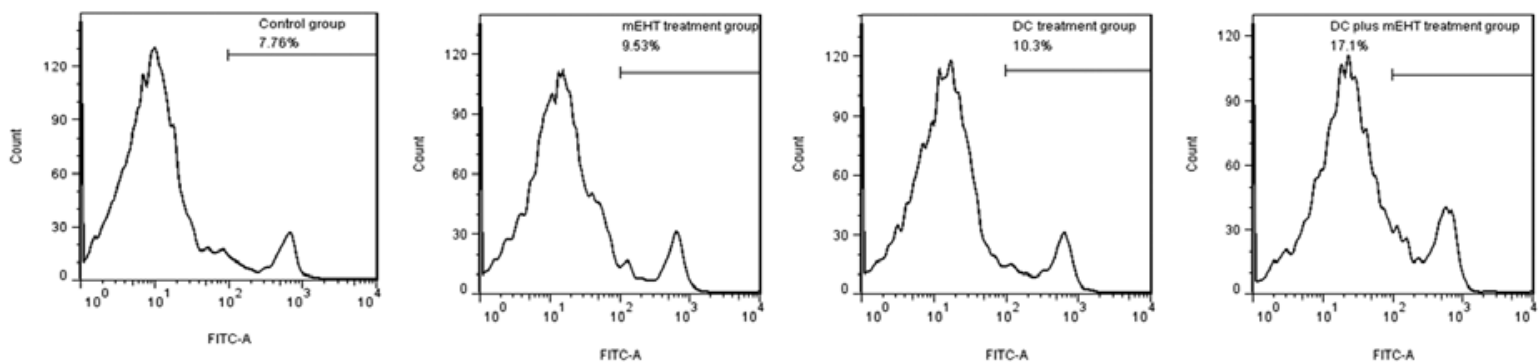

Figure 3. Flow cytometric analysis of $\mathrm{CD}^{+}$and $\mathrm{CD} 8^{+} \mathrm{T}$ cells in the tumor draining lymph nodes of mice treated with dendritic cells (DCs) and modulated electro-hyperthermia (mEHT), either alone or in combination. A significant increment in the population of $\mathrm{CD}^{+}$and $\mathrm{CD} 8^{+} \mathrm{T}$ cells was observed in the $\mathrm{DC}$ plus mEHT treatment group.

whereas the mean volume of the tumors in the DC and the mEHT treatment group was only $99.84 \pm 9.21 \mathrm{~mm}^{3}$. The tumor growth rate was obviously slower in the DC and mEHT treatment group compared to the control group $(\mathrm{P}<0.05)$. The mean chest tumor volumes in the DC-only treatment group and the mEHT-only treatment group were $396.32 \pm 58.52$ and $152.78 \pm 19.12 \mathrm{~mm}^{3}$, respectively, both of which were also significantly smaller $(\mathrm{P}<0.05)$ than the mean tumor volume in the control group (Fig. 2B). This phenomenon was thought to be the abscopal effect.

$C D 3^{+}$and $C D 8^{+} T$ cells in the TDLNs. We investigated whether the intratumoral injection of DCs in addition to mEHT can elicit a systemic antitumor effect in vivo by examining the populations of $\mathrm{CD}^{+}$and $\mathrm{CD} 8^{+} \mathrm{T}$ cells in the TDLNs. As shown in Fig. 3, the $\mathrm{CD}^{+}$population in the TDLNs of the mice treated with DCs plus mEHT was $\sim 5$ times larger compared with this population in the TDLNs of the control group, and the $\mathrm{CD} 8^{+}$population in the TDLNs of the mice treated with DCs plus mEHT was $\sim 2$ times larger. This suggests that the systemic antitumor activity that was evoked by DC plus mEHT treatment can be a sensitizer of a DC-based immunoresponse. Furthermore, we also evaluated whether mEHT alone has the possibility of inducing $\mathrm{CD}^{+}$and $\mathrm{CD} 8^{+} \mathrm{T}$ cells. We found that mEHT alone could increase that population. This may be one of the pieces of evidence that can explain the mechanism of the absocopal effects.

Immunostaining of the tumors. For the immunostaining, the tumor tissues were examined for CD8, S100 and Foxp3 protein expression. The IOD was calculated as the number of positive cells expressing the protein. We found that in the DC plus $\mathrm{mEHT}$ treatment group, a greater number of cells were positive for CD8 than the numbers in the other groups (Fig. 4) (control group: 10,607.17 $\pm 1,193.27$; DC treatment group: $20,775.26 \pm 1,56.91$; mEHT treatment group: $30,893.19 \pm 2,067.52$; DC and mEHT treatment group: $63,028.26 \pm 8,281.73 ; \mathrm{P}<0.05)$. This indicates that a greater number of cytotoxic lymphocytes were stimulated to attack the tumor cells following the combined treatment. Similar findings were noted for S100, a DC marker, with a significantly greater number of S100-positive cells noted in the DC plus mEHT treatment group compared with the other groups (Fig. 4) (control group: 25,944.49 $\pm 2,084.24$; DC treatment group: $30,108.26 \pm 1,827.21$; mEHT treatment group: 27,685.48 $\pm 1,373.36$; DCs and mEHT treatment group: $53,118.53 \pm 6,680.50, \mathrm{P}<0.05)$. This indicates that a greater number of DCs were induced to take part in the immune reaction against the tumor. Unlike these other markers, the extent of staining of Foxp3, a marker and determinant of regulatory $\mathrm{T}$ (Treg) cells, was significantly higher in the control group compared with the other 3 groups (Fig. 4) (control group: 42,773.29 $\pm 1,461.24$; DC treatment group: 31,209.38 $\pm 1,928.48$; mEHT treatment group: $25,394.31 \pm 1,323.39$; DC and mEHT treatment group: $17,496.13 \pm 1,018.26, \mathrm{P}<0.05)$, suggesting that the immunosuppression by Treg cells in the treated tissue was reduced by mEHT.

$R T-P C R$. RT-PCR was used to analyze the cDNA reversetranscribed RNA of the chest tumors from different groups. As shown in Fig. 5, regarding the expression of GP96, 6-fold increase was observed in the DC plus mEHT treatment group compared to the control group. In addition, the expression of GP96 exhibited a 2-fold increase in the DC treatment group and a 1-fold increased in the mEHT treatment group. Based 


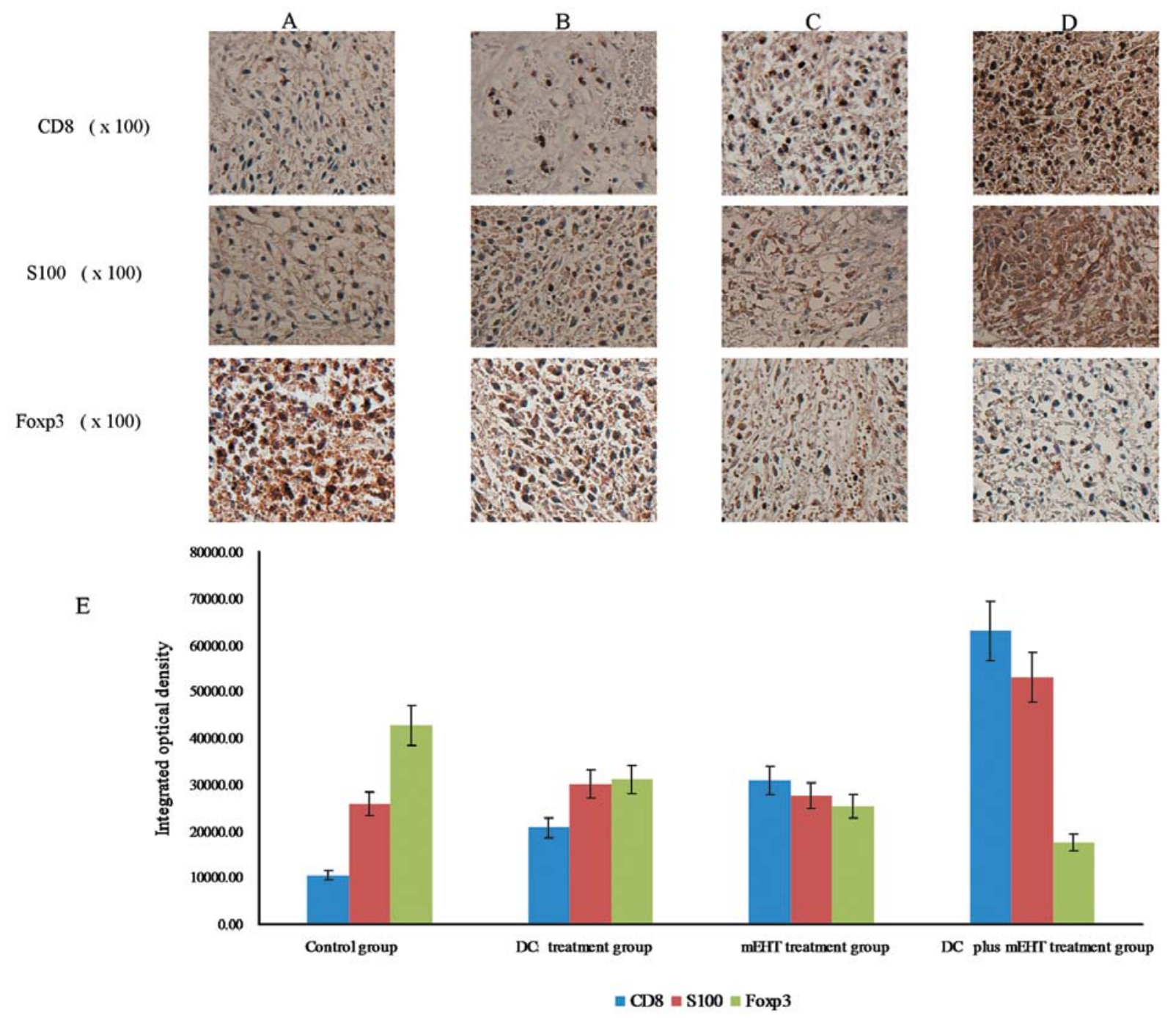

Figure 4. Changes in CD8, S100 and Foxp3 expression in the chest tumor tissues of the different groups (original magnification x100). (A) Control group. (B) Dendritic cell (DC) treatment group. (C) Modulated electro-hyperthermia (mEHT) treatment group. (D) DC plus mEHT treatment group. (E) The intergrated optical density (IOD) values were examined between the different groups separately.

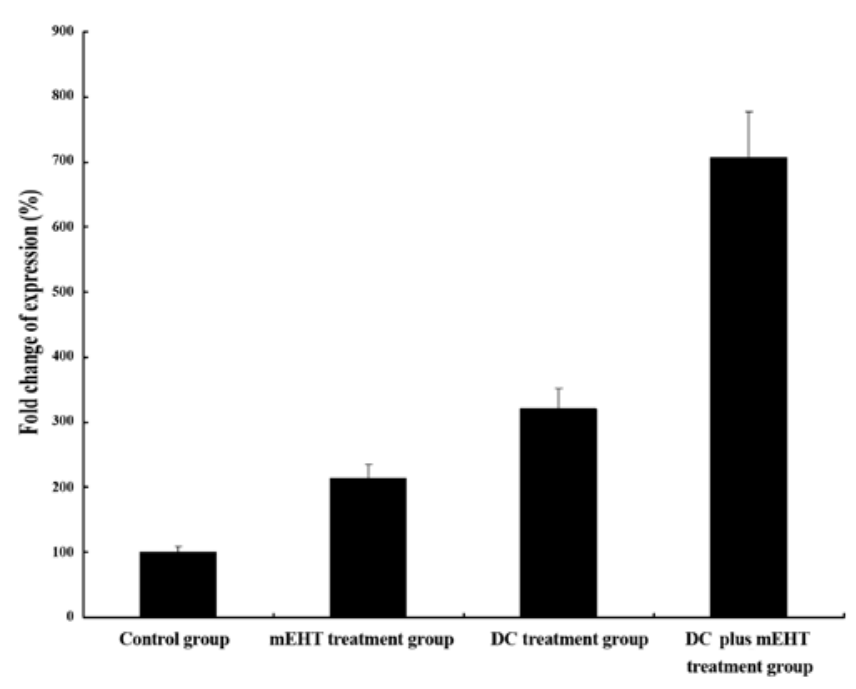

Figure 5. Analysis of GP96 expression in the different groups. Dendritic cell (DC) plus mEHT treatment upregulated the expression of GP96 compared to the other groups. on the data, the GP96 gene was amplified in the treatment groups compared to the control group. This may at least in part explain the mechanism underlying the abscopal effect.

\section{Discussion}

Head and neck and esophageal cancer are still among the most deadly malignant neoplasms, particularly in the Asian populaton. These cancers have the tendency to metastasize easily to lymph nodes $(14,15)$. Their prognosis is poor and unsatisfactory even with current treatments, as lymph node metastases and distant organ metastases occur frequently. The development of effective treatments is urgent.

Hyperthermia is a type of cancer treatment in which tissue is exposed to a high temperature. Previous studies have shown that high temperatures can damage and kill cancer cells, usually with minimal injury to normal tissues. mEHT which is also described as 'oncothermia' in Europe is a personalized treatment using energy delivery to the targeted tumor (9). This treatment concentrates the absorbed energy to intercellular electrolytes (8). According to clinical studies of 
mEHT, the application of mEHT has good clinical outcome and makes a stable basis for clinical applications in various advanced primary and metastatic malignancies, such as bone (metastatic) (16), breast (17) and malignant gliomas (18). In the present study, as shown in Fig. 1, only the tumor underwent an increase in temperature during $\mathrm{mEHT}$, without any increase in rectal temperature. The temperature of the center of the tumor is higher than the temperature of the tumor surface. This differential temperature rise is achieved through the precise surface control and moderate power usage in $\mathrm{mEHT}$ that enables the temperature to be finely regulated (19).

In the present study, we found that treatment of the tumors on the leg of mice resulted in the concurrent growth inhibition of the distant tumors on the chest. This phenomenon is known as the abscopal effect and is defined as a reaction following irradiation but occurring outside the zone of actual radiation absorption. It was first described more than 60 years ago by Dr R.H. Mole, of Britain's Medical Research Council (20). He was the first to use the term 'abscopal effect,' where 'abscopal' is derived from the Latin prefix $a b$, meaning 'away from,' and the Greek word skopos, meaning 'target.' Multiple case studies describing an abscopal effect observed after radiotherapy have been published for a variety of malignancies, including lymphoma, papillary adenocarcinoma, melanoma, adenocarcinoma of the esophagus, chronic lymphocytic leukemia and hepatocellular carcinoma (21-25).

The mechanism that underlies the abscopal effect remains unclear, although studies in mice suggest that it may depend upon activation of the immune system, and is mediated by T cells (7). Our findings also suggest that distant tumor regression was mediated by an immune response (via $\mathrm{CD}^{+}$and $\mathrm{CD} 8^{+}$cells) activated by mEHT and DCs. The combination of mEHT and DCs produced tumor antigens by apoptosis and/or necrosis of tumor cells, and also induced the abscopal effect against SCCVII cells. Moreover, direct intratumoral administration of DCs are an important strategy. DCs can be pulsed with a wide range of antigens and they are efficient for the direct trafficking into tumors. This means that the trafficking loss of DCs administered is minimized (26-28).

In the present research, we found that mEHT enhanced the expression of GP96 in vivo. As shown in Fig. 5, mEHT treatment had the ability to induce the expression of GP96. The product of this gene plays an important role in the uptake of antigens by DCs. The tumor antigens form a complex with GP96, which is then taken up by DCs through the GP96 receptor in a process mediated by toll-like receptors 2 and 4 . This leads to DC maturation, enabling them in turn to induce CD8 ${ }^{+}$CTL activation (29-33). In the present study, we found that the upregulation of GP96 by mEHT led to an increase in the number of $\mathrm{CD} 8^{+} \mathrm{CTLs}$ in the TDLNs. This suggests that DCs pulsed with mEHT exerted a stronger cytotoxic effect leading to tumor growth inhibition in vivo, via an increase in the level of GP96 protein. Thus, one key molecule, by which the abscopal effect induces systemic antitumor immune reaction, may be GP96. As others have reported, GP96 potentially boosts the activity of the systemic antitumor effect by these CTLs, and this mechanism could be one explanation for the abscopal effect (34-36).

In a case study from the Memorial Sloan-Kettering Cancer Center in New York, changes in a metastatic melanoma patient's immune system were measured over the course of treatment (1). Changes in tumor-directed antibody levels and immune cell populations were found to coincide with an abscopal effect. These findings support the idea that radiation may help stimulate the immune system to fight cancer. We believe that local hyperthermia induced both, direct and abscopal antitumor effects, and that the latter may have been the result of a systemic effect of hyperthermia. We found that there was a significantly greater number of CD8- and S100-positive cells after combined DC and mEHT treatment. This indicates that additional cytotoxic lymphocytes were stimulated to attack the tumor cells. Furthermore, there were fewer Foxp3-positive cells after this treatment, suggesting that the immune-suppression mediated by Treg cells in the treated tissue was reduced. Correspondingly, combined treatment with DCs plus mEHT induced a more pronounced antitumor effect against distant non-treated tumors compared with the leg tumors treated with DCs alone. This is particularly important for cancer treatment, as the combination of DCs and mEHT exerted a strong abscopal effect against the distant non-treated tumors.

Our findings suggest that the combination of hyperthermia with an intratumoral injection of DCs can induce whole body antitumor effects. Both DC and mEHT therapy are non-invasive, and therefore, this treatment model can be safely adapted for treating patients. Based on our findings, the combination of direct intratumoral administration of DCs and mEHT can be a feasible future treatment, and warrants further studies, including human clinical trials.

\section{Acknowledgements}

This study was supported by the JSPS KAKENHI (grant no. 24501328).

\section{References}

1. Postow MA, Callahan MK, Barker CA, et al: Immunologic correlates of the abscopal effect in a patient with melanoma. $\mathrm{N}$ Engl J Med 366: 925-931, 2012.

2. Perego D and Faravelli A: Unexpected consequence of splenectomy in composite lymphoma. The abscopal effect. Haematologica 85: 211, 2000.

3. Okuma K, Yamashita H, Niibe Y, Hayakawa K and Nakagawa K: Abscopal effect of radiation on lung metastases of hepatocellular carcinoma: a case report. J Med Case Rep 5: 111, 2011.

4. Macklis RM, Mauch PM, Burakoff SJ and Smith BR: Lymphoid irradiation results in long-term increases in natural killer cells in patients treated for Hodgkin's disease. Cancer 69: 778-783, 1992.

5. Lugade AA, Moran JP, Gerber SA, Rose RC, Frelinger JG and Lord EM: Local radiation therapy of B16 melanoma tumors increases the generation of tumor antigen-specific effector cells that traffic to the tumor. J Immunol 174: 7516-7523, 2005.

6. Lee Y, Auh SL, Wang Y, et al: Therapeutic effects of ablative radiation on local tumor require $\mathrm{CD} 8{ }^{+} \mathrm{T}$ cells: changing strategies for cancer treatment. Blood 114: 589-595, 2009.

7. Demaria S, Ng B, Devitt ML, et al: Ionizing radiation inhibition of distant untreated tumors (abscopal effect) is immune mediated. Int J Radiat Oncol Biol Phys 58: 862-870, 2004.

8. Andocs G, Renner H, Balogh L, Fonyad L, Jakab C and Szasz A: Strong synergy of heat and modulated electromagnetic field in tumor cell killing. Strahlenther Onkol 185: 120-126, 2009.

9. Andocs G, Szasz O, Szasz A: Oncothermia treatment of cancer: from the laboratory to clinic. Electromagn Biol Med 28: 148-165, 2009.

10. Khurana D, Martin EA, Kasperbauer JL, et al: Characterization of a spontaneously arising murine squamous cell carcinoma (SCC VII) as a prerequisite for head and neck cancer immunotherapy. Head Neck 23: 899-906, 2001. 
11. Lutz MB, Kukutsch N, Ogilvie AL, et al: An advanced culture method for generating large quantities of highly pure dendritic cells from mouse bone marrow. J Immunol Methods 223:77-92, 1999.

12. Workman P, Aboagye EO, Balkwill F, et al: Guidelines for the Welfare and Use of Animals in Cancer Research. Br J Cancer 102: 1555-1577, 2010.

13. Xavier LL, Viola GG, Ferraz AC, et al: A simple and fast densitometric method for the analysis of tyrosine hydroxylase immunoreactivity in the substantia nigra pars compacta and in the ventral tegmental area. Brain Res Brain Res Protoc 16: 58-64, 2005.

14. Bollschweiler E, Baldus SE, Schroder W, et al: High rate of lymph-node metastasis in submucosal esophageal squamous-cell carcinomas and adenocarcinomas. Endoscopy 38: 149-156, 2006

15. Mayinger B, Horner P, Jordan M, et al: Light-induced autofluorescence spectroscopy for the endoscopic detection of esophageal cancer. Gastrointest Endosc 54: 195-201, 2001.

16. Bogovic J, Douwes F, Muravjov G and Istomin J: Posttreatment histology and microcirculation status of osteogenic sarcoma after a neoadjuvant chemo- and radiotherapy in combination with local electromagnetic hyperthermia. Onkologie 24: 55-58, 2001.

17. Feyerabend T, Wiedemann GJ, Jager B, Vesely H, Mahlmann B and Richter E: Local hyperthermia, radiation, and chemotherapy in recurrent breast cancer is feasible and effective except for inflammatory disease. Int J Radiat Oncol Biol Phys 49: 1317-1325, 2001.

18. Fiorentini G, Giovanis P, Rossi S, et al: A phase II clinical study on relapsed malignant gliomas treated with electro-hyperthermia. In Vivo 20: 721-724, 2006.

19. Hegyi G, Szigeti GP and Szasz A: Hyperthermia versus oncothermia: cellular effects in complementary cancer therapy. Evid Based Complement Alternat Med 2013: 672873, 2013.

20. Mole RH: Whole body irradiation; radiobiology or medicine? Br J Radiol 26: 234-241, 1953.

21. Nobler M: The abscopal effect in malignant lymphoma and its relationship to lymphocyte circulation. Radiology 93: 410-412, 1969.

22. Rees GJ: Abscopal regression in lymphoma: a mechanism in common with total body irradiation? Clin Radiol 32: 475-480, 1981.

23. Rees GJ and Ross CM: Abscopal regression following radiotherapy for adenocarcinoma. Br J Radiol 56: 63-66, 1983.
24. Sham RL: The abscopal effect and chronic lymphocytic leukemia. Am J Med 98: 307-308, 1995.

25. Ohba K, Omagari K, Nakamura T, et al: Abscopal regression of hepatocellular carcinoma after radiotherapy for bone metastasis. Gut 43: 575-577, 1998

26. Schmidt T, Ziske C, Marten A, et al: Intratumoral immunization with tumor RNA-pulsed dendritic cells confers antitumor immunity in a C57BL/6 pancreatic murine tumor model. Cancer Res 63: 8962-8967, 2003.

27. Candido KA, Shimizu K, McLaughlin JC, et al: Local administration of dendritic cells inhibits established breast tumor growth: implications for apoptosis-inducing agents. Cancer Res 61:228-236, 2001.

28. Triozzi PL, Khurram R, Aldrich WA, Walker MJ, Kim JA and Jaynes S: Intratumoral injection of dendritic cells derived in vitro in patients with metastatic cancer. Cancer 89:2646-2654, 2000.

29. Binder RJ and Srivastava PK: Essential role of CD91 in re-presentation of gp96-chaperoned peptides. Proc Natl Acad Sci USA 101: 6128-6133, 2004.

30. Facciponte JG, Wang XY, MacDonald IJ, et al: Heat shock proteins HSP70 and GP96: Structural insights. Cancer Immunol Immunother 55: 339-346, 2006.

31. Demine R and Walden P: Testing the role of gp96 as peptide chaperone in antigen processing. J Biol Chem 280: 17573-17578, 2005.

32. Ramirez SR, Singh-Jasuja H, Warger T, et al: Glycoprotein 96-activated dendritic cells induce a CD8-biased T cell response. Cell Stress Chaperones 10: 221-229, 2005.

33. Segal BH, Wang XY, Dennis CG, et al: Heat shock proteins as vaccine adjuvants in infections and cancer. Drug Discov Today 11: 534-540, 2006.

34. Akutsu Y, Matsubara H, Urashima T, et al: Combination of direct intratumoral administration of dendritic cells and irradiation induces strong systemic antitumor effect mediated by GRP94/ gp96 against squamous cell carcinoma in mice. Int J Oncol 31: 509-515, 2007.

35. Wang XH, Qin Y, Hu MH and Xie Y: Dendritic cells pulsed with gp96-peptide complexes derived from human hepatocellular carcinoma (HCC) induce specific cytotoxic T lymphocytes. Cancer Immunol Immunother 54: 971-980, 2005.

36. Liu S, Wang H, Yang Z, et al: Enhancement of cancer radiation therapy by use of adenovirus-mediated secretable glucoseregulated protein 94/gp96 expression. Cancer Res 65: 9126-9131, 2005. 